\title{
Principais fatores de risco associados ao óbito fetal: revisão integrativa
}

\author{
Main risk factors associated with fetal death: integrative review \\ Principales factores de riesgo asociados con la muerte fetal: revisión integradora
}

Thais de Albuquerque Corrêa ${ }^{1 *}$, Elisama da Paz Oliveira Lima ${ }^{1}$, Aniely Tavares da Silva ${ }^{1}$, Luciana da Silva Barreto ${ }^{1}$, Renata Paula Pereira da Silva ${ }^{1}$, Clara de Holanda Braga ${ }^{1}$, Laise Risalva Farias Gouveia da Silva ${ }^{1}$, Camila Dias da Silva Barros ${ }^{1}$, Edjane Maria da Silva ${ }^{2}$, Rutheanne Melo de Siqueira ${ }^{1}$.

\section{RESUMO}

Objetivo: Identificar na literatura as principais causas associadas ao óbito fetal. Métodos: Trata-se de uma revisão integrativa de literatura com busca na base de dados da BIREME (Biblioteca regional de Medicina) utilizando os descritores: "Morte fetal", "fetal death"; "Pregnancy High Risk" e "Gravidez de alto risco". Os critérios de inclusão consistiram em artigos publicados nos últimos cinco anos, com texto completo disponível. Após a seleção dos critérios de inclusão e exclusão, foram selecionados 9 artigos lidos na íntegra e suas informações foram compactadas e organizadas. Resultados: Prevaleceram as causas mais presentes de óbitos fetais: transtorno materno hipertensivo, complicações da placenta, placenta prévia e diabetes. Esses motivos de óbito fetal foram associados às gestantes com idade acima de 35 anos, além de possuírem alguns fatores de risco, tais como: baixo nível socioeconômico, tabagismo, uso de drogas, obesidade e gestantes que tiveram uma má qualidade da assistência pré-natal e escassez no acompanhamento intraparto. Considerações finais: E considerável ressaltar a importância dos indicadores de mortalidade perinatal, que indica o estado de saúde e qualidade prestada a assistência as gestantes.

Palavras-chave: Morte fetal, Gestantes, Gravidez de alto risco.

\begin{abstract}
Objective: To identify in the literature the main causes associated with fetal death. Methods: It is an integrative literature review with a search in the database of BIREME (Regional Library of Medicine) using the descriptors: "Fetal death", "fetal death", "Pregnancy High Risk" and "High Risk Pregnancy". Inclusion criteria consisted of articles published in the last five years, with full text available. After selecting the inclusion and exclusion criteria, 9 articles read in full were selected and their information was compacted and organized. Results: The most prevalent causes of fetal deaths prevailed: hypertensive maternal disorder; placental complications; placenta previa and diabetes. These reasons for fetal death were associated with pregnant women over the age of 35 , in addition to having some risk factors, such as: low socioeconomic status, smoking, drug use, obesity and pregnant women who had poor quality prenatal care and scarcity in intrapartum follow-up. Final considerations: It is considerable to emphasize the importance of perinatal mortality indicators, which indicate the health status and quality of care provided to pregnant women.
\end{abstract}

Key words: Fetal death, Pregnant women, Pregnancy high risk.

\section{RESUMEN}

Objetivo: Identificar en la literatura las principales causas asociadas a la muerte fetal. Métodos: Se trata de una revisión integradora de la literatura con búsqueda en la base de datos de BIREME (Biblioteca Regional de Medicina) utilizando los descriptores: "Muerte fetal", "muerte fetal", "Embarazo de alto riesgo" y "Embarazo de alto riesgo". Los criterios de inclusión consistieron en artículos publicados en los últimos cinco años, con texto completo disponible Luego de seleccionar los criterios de inclusión y exclusión, se seleccionaron 9 artículos leídos en su totalidad y su información fue compactada y organizada. Resultados: Predominaron las causas más prevalentes de muerte fetal: trastorno materno hipertensivo; complicaciones placentarias;

\footnotetext{
${ }^{1}$ Faculdade Pernambucana de Saúde (FPS), Recife - PE. *E-mail: thaiscorrea_10@hotmail.com

${ }^{2}$ Faculdade de Ciências Humanas de Olinda (FACHO), Olinda - PE.
} 
placenta previa y diabetes. Estos motivos de muerte fetal se asociaron con mujeres embarazadas mayores de 35 años, además de tener algunos factores de riesgo, como: nivel socioeconómico bajo, tabaquismo, consumo de drogas, obesidad y mujeres embarazadas que tenían atención prenatal de mala calidad. y escasez en el seguimiento intraparto. Consideraciones finales: Es considerable destacar la importancia de los indicadores de mortalidad perinatal, que indican el estado de salud y la calidad de la atención brindada a las gestantes.

Palabras clave: Muerte fetal, Mujeres embarazadas, Embarazo de alto riesgo.

\section{INTRODUÇÃO}

A gravidez é um acontecimento natural, fisiológico e enérgico, onde, na maior parte das ocasiões acontece sem problemas e contrariedades (ANTUNES MB, et al., 2017). Este período da vida da mulher é uma fase em que ocorrem alterações profundas em seu estilo de vida, causando mudanças não apenas na vida pessoal, mas também na vida do casal e de toda a família, além de ser uma etapa de preparo físico e psicológico (COUTINHO EDC, et al., 2014). Mesmo com toda atenção e prudência, há casos de gestantes que passam por complicações de saúde, ou até mesmo desenvolvem doenças ao decorrer da gestação (ANTUNES MB, et al., 2017). Por isso, é importante um acompanhamento adequado e uma assistência de qualidade às mulheres que estão gestantes o mais rápido possível para que isso possa, de alguma forma, conseguir evitar prováveis distúrbios. Assim, descrever o perfil epidemiológico das gestantes de alto risco podem movimentar investigações importantes e aperfeiçoar ações preventivas imediatas para tais complicações (DALLA COSTA L, et al., 2016).

Quanto aos óbitos fetais, acontecem cerca de 2,6 milhões por ano no mundo com prevalência nos países de baixa e média renda e, infelizmente, os mesmos permanecem ignorados nas políticas e programas públicos de saúde, tanto no nível internacional quanto local. Já nos países de alta renda, a taxa de natimortos diminuiu bastante devido à melhoria no cuidado intraparto e antibióticos eficazes para tratar infecções e outros tipos de comorbidades (PAGE JM e SILVER RM, 2017).

Diversas indagações dirigidas e originadas no Brasil destacaram e evidenciaram a análise dos determinados pontos: inclinação e predisposição da mortalidade fetal, qualidade das informações que foram registradas na Declaração de óbito e as causas e os fatores associados à mortalidade fetal. Essas indagações apontaram uma diminuição na mortalidade fetal na última década, havendo uma redução de $3,7 \%$ na taxa de mortalidade fetal entre os anos de 1996 e 2012, contudo a mesma permanece alta (MOURA BLA, 2017). No Brasil, entre os anos de 2000 à 2016, foi observada uma taxa de 5,3 óbitos fetais por mil nascimentos (GBD, 2016; MORTALITY COLLABORATORS, 2017).

Para o Ministério da Saúde, o óbito fetal acontece quando há perda do produto da concepção após a 22ํㅡ semana de gravidez ou quando há perda de fetos com peso igual ou superior a $500 \mathrm{~g}$. Porém, com o propósito de contribuir nas comparações internacionais, a Organização Mundial de Saúde (OMS) aplicou a definição de óbito fetal tardio aquele ocorrido com 28 ou mais semanas de gestação (MOURA BLA, 2017).

Identificar gestações com risco aumentado é primordial para reduzir a natimortalidade. Muitas características maternas como obesidade, idade materna avançada (idade > 35 anos), tabagismo, hipertensão crônica e diabetes pré-gestacional são associadas a uma chance elevada de morte fetal. Segundo o Ministério da Saúde, condições clínicas preexistentes também podem ser marcadores importantes e fatores de risco significativos, tais como: cardiopatias, nefropatias, neoplasias, epilepsia, doenças autoimunes, ginecopatias, hemopatias, e, também, doenças infecciosas (PAGE JM e SILVER RM, 2017; ESTRATÉGICAS, 2010).

As alterações fisiológicas que ocorrem na gravidez são extremamente importantes na investigação de possíveis doenças ou problemas que acarretem no óbito fetal, visto que, essa eventualidade é um acontecimento psicologicamente traumático para a mulher e também para a sua família, podendo ocasionar sérios efeitos em longo prazo para a gestante. Visto isso, as causas de morte são importantes para a compreensão e prevenção dos óbitos fetais. Este artigo tem como objetivo sintetizar os estudos que envolvem 
o problema "óbito fetal" para que possam ser identificados seus principais fatores de risco. Isto contribuirá para que outras pesquisas sejam realizadas a partir dos achados aqui apresentados, podendo identificar lacunas na literatura além de alertar a sociedade e comunidade científica sobre como prevenir, se possível, o óbito fetal.

\section{MÉTODOS}

O método escolhido para responder ao objetivo colocado foi a revisão integrativa da literatura. Consiste em um método vasto de análise de pesquisas a fim de fundir conhecimento em relação a determinado tema. A elaboração de um estudo dessa natureza perpassa por cinco etapas, sendo elas: identificação do problema e elaboração da questão norteadora; busca dos estudos na literatura; avaliação de dados encontrados nos estudos; análise de dados com síntese e conclusões destes e apresentação da revisão integrativa (HOPIA H, et al., 2016).

A busca dos estudos foi realizada no mês de julho de 2019, onde, os mesmos foram selecionados na base de dados eletrônicos Biblioteca Virtual em Saúde (BVS), no qual, os artigos escolhidos faziam parte da base de dados eletrônica LILACS (Literatura Latino-Americana e do Caribe em Ciências da Saúde), Medline (Literature Analysis and Retrieval System Online) que, em português, significa "Sistema Online de Busca e Análise de Literatura Médica" e IBECS (Índice Bibliográfico Espanhol de Ciências de Saúde).

Para dar continuidade com a busca, utilizaram-se Descritores em Ciências da Saúde (DeCS) para as bases de dados em língua portuguesa e inglesa. Também foi colocado o operador boleano AND para interligar os descritores da seguinte forma: "Morte fetal $A N D$ Fetal death $A N D$ Pregnancy High Risk $A N D$ Gravidez de alto risco". Os critérios de inclusão consistiram em artigos publicados nos últimos cinco anos (de 2014 a 2019) e com texto completo disponível. Foram desconsiderados artigos repetidos e estudos que não corresponderam à temática de acordo com o objetivo da revisão.

A busca inicial na base de dados (BIREME) a partir da utilização dos descritores selecionados (Morte fetal; fetal death; Pregnancy High Risk; Gravidez de alto risco) atingiu um total de 349 artigos. Em seguida, utilizaram-se os filtros oferecidos pela mesma (acesso disponível ao texto completo e publicação nos últimos cinco anos - De 2014 a 2019), o que totalizou em 31 artigos, assim, excluindo 318 artigos por não responderem ao objetivo proposto. Também foram excluídos os estudos repetidos, onde a amostra resultou em 30 artigos. O processo de busca e seleção dos estudos está representado na Figura 1.

Figura 1 - Fluxograma da seleção dos estudos.

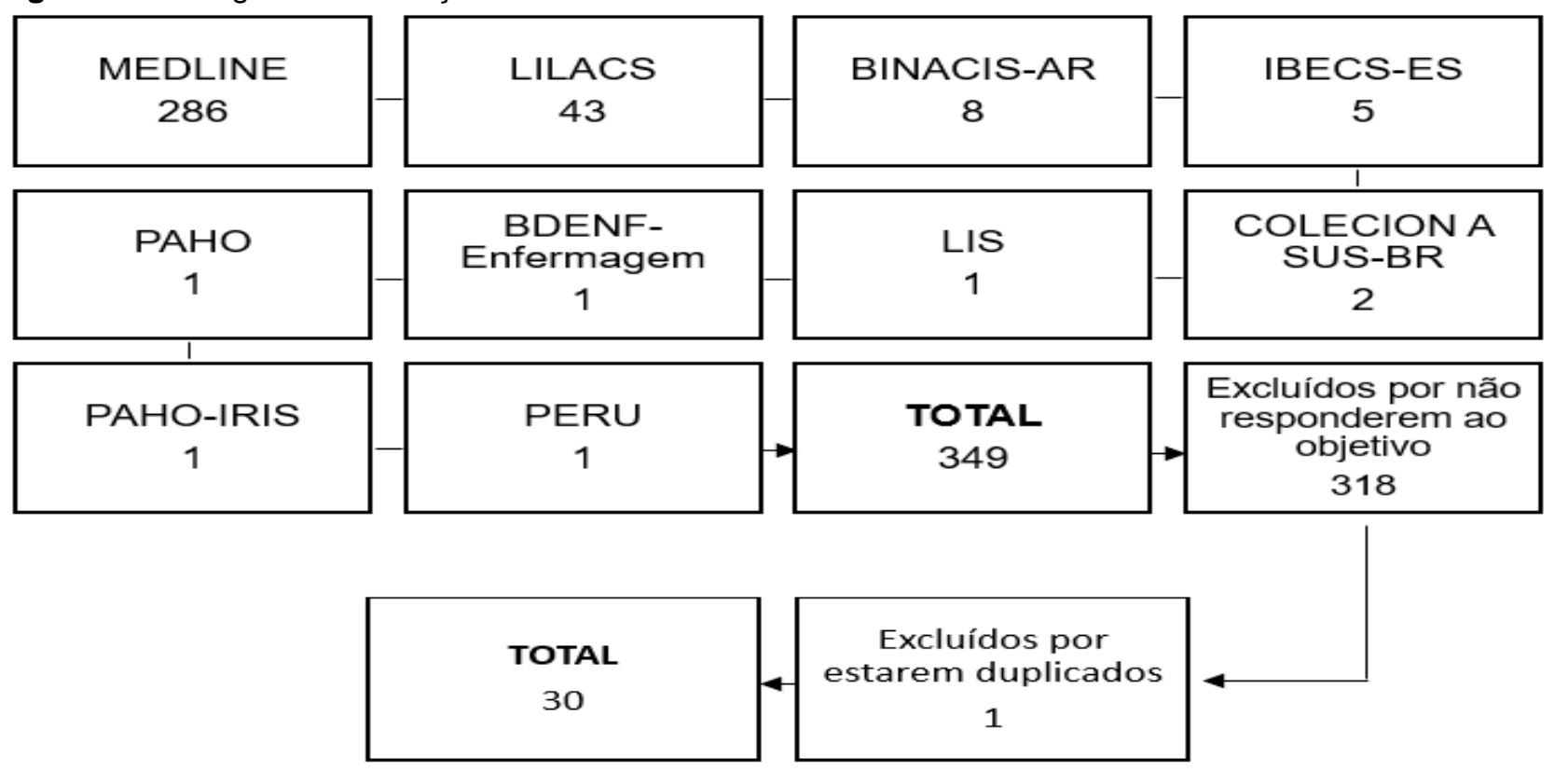

Fonte: Corrêa TA, et al., 2021. 
Por fim, foram selecionados 9 artigos a partir da leitura do título e resumos (Figura 2).

Figura 2 - Fluxograma dos estudos selecionados a partir da leitura dos títulos e resumos.

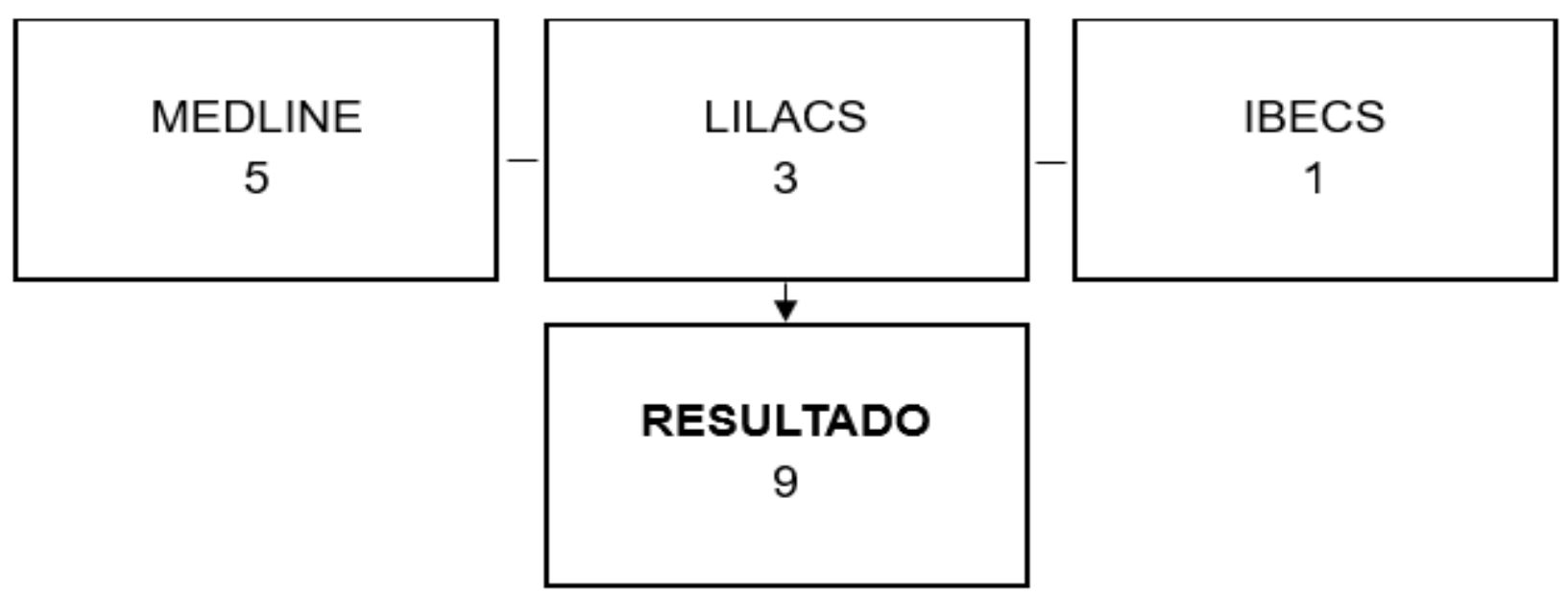

Fonte: Corrêa TA, et al., 2021.

A análise e discussão dos resultados pautaram-se na literatura nacional e internacional sobre os principais fatores de risco relacionados ao óbito fetal.

\section{RESULTADOS E DISCUSSÃO}

Para organizar e analisar melhor os estudos foram elaborados em uma planilha criada no Software Microsoft World contendo os seguintes itens: título do artigo, ano de publicação, base de dados, periódico e idioma. Com ela, foi possível visualizar melhor todos os artigos que foram utilizados (Tabela 3). 
Tabela 3 - Distribuição das publicações escolhidas para análise segundo títulos, ano de publicação, base de dados, periódico e idioma.

\begin{tabular}{|c|c|c|c|c|}
\hline Título & Ano & Base de dados & Idioma & Principais resultados \\
\hline $\begin{array}{l}\text { Aspirine: indications et utilization durant la } \\
\text { grossesse. }\end{array}$ & 2017 & MEDLINE & Francês & $\begin{array}{l}\text { Previu-se que a utilização de aspirina em mulheres grávidas pode ajudar a prevenir } \\
\text { a doença vascular placentária em pacientes em risco. Vários estudos provaram a } \\
\text { sua eficácia na prevenção da pré-eclâmpsia. }\end{array}$ \\
\hline Interventions to prevent stillbirth. & 2017 & MEDLINE & Inglês & $\begin{array}{l}\text { Condições médicas maternas como diabetes mellitus foram alguns dos primeiros } \\
\text { fatores de risco reconhecidos para natimortalidade. Distúrbios hipertensivos afetam } \\
7 \text { a } 10 \% \text { das gestações. Hipertensão crônica, hipertensão gestacional e pré- } \\
\text { eclâmpsia são prováveis causa de morte em } 10 \% \text { dos partos prematuros anteparto. }\end{array}$ \\
\hline $\begin{array}{l}\text { Gestantes usuárias do Sistema Único de } \\
\text { Saúde no município de São Paulo: } \\
\text { desfechos de uma coorte de dados } \\
\text { secundários. }\end{array}$ & 2017 & LILACS & Português & $\begin{array}{l}\text { Anormalidades genéticas e morbidades maternas (hipertensão, diabetes, } \\
\text { patologias placentárias) são relatadas como fatores associados ao óbito fetal. }\end{array}$ \\
\hline $\begin{array}{l}\text { Síndrome hipertensiva e resultados } \\
\text { perinatais em gestação de alto risco. }\end{array}$ & 2017 & LILACS & Português & $\begin{array}{c}\text { As Síndromes Hipertensivas manifestam-se como a segunda causa de morte } \\
\text { materna em todo o mundo, sendo superada apenas pelas hemorragias, elevando, } \\
\text { assim, o número de óbitos fetais. }\end{array}$ \\
\hline $\begin{array}{l}\text { ACR Appropriateness Criteria } \\
\text { Assessment of Fetal Well-Being. }\end{array}$ & 2016 & MEDLINE & Inglês & $\begin{array}{l}\text { A vigilância anteparto é recomendada para avaliar o estado fetal em gestações de } \\
\text { alto risco. Em gravidezes prematuras com oligoidramnios ou polidrâmnio, a } \\
\text { vigilância em série é garantida após uma avaliação minuciosa por possíveis } \\
\text { causas. }\end{array}$ \\
\hline $\begin{array}{l}\text { Placenta previa and immediate outcome } \\
\text { of the term offspring. }\end{array}$ & 2016 & MEDLINE & Inglês & $\begin{array}{l}\text { O tempo de parto (prematuro versus termo) é o provável preditor principal de } \\
\text { sobrevida neonatal e desfecho em gestações com placenta prévia. }\end{array}$ \\
\hline $\begin{array}{c}\text { Anticardiolipin antibodies and recurrent } \\
\text { spontaneous abortion in Brazilian women. }\end{array}$ & 2015 & LILACS & Inglês & $\begin{array}{l}\text { A prevalência dos anticorpos anticardiolipina é alta em uma história de aborto } \\
\text { espontâneo recorrente. }\end{array}$ \\
\hline $\begin{array}{l}\text { Doppler velocimetry of the uterine artery } \\
\text { blood flow and risk of perinatal death in } \\
\text { preeclamptic patients. }\end{array}$ & 2014 & IBECS & Espanhol & $\begin{array}{l}\text { Foi analisado que, o padrão anormal da velocimetria de fluxo das artérias uterinas } \\
\text { nas artérias pré-eclâmpticas entre } 32 \text { e } 37 \text { semanas está associado ao risco de } \\
\text { morte perinatal. }\end{array}$ \\
\hline $\begin{array}{l}\text { Magnesium supplementation in } \\
\text { pregnancy. }\end{array}$ & 2014 & MEDLINE & Inglês & $\begin{array}{c}\text { Não há efeitos estatisticamente significativos da suplementação de magnésio sobre } \\
\text { a frequência de mortalidade perinatal ou de crianças pequenas para a idade } \\
\text { gestacional quando comparados com placebo ou nenhum tratamento. }\end{array}$ \\
\hline
\end{tabular}

Fonte: Corrêa TA, et al., 2021. 
O baixo número de estudos que integraram a revisão integrativa remete à possível escassez dos pesquisadores em se interessarem ao assunto referido e colocá-lo como algo muito importante a ser estudado, tendo em vista que tal temática é de extrema relevância não só para as mães, mas também para a população, estudantes e profissionais da área de saúde. A predominância de estudos publicados na língua inglesa reflete o fato de ser esse um idioma amplamente disseminado no mundo. A taxa de mortalidade fetal (TMF) é um indicativo de qualidade da assessoria oferecida à gestante, inclusive no momento do parto, onde, é apresentada pelo valor de óbitos fetais por 1.000 nascimentos totais em determinada população habitante de um dado local e ano. A definição da TMF no Brasil é um transtorno que deve ser superado, pois, alguns estudos apontam uma certa vulnerabilidade na qualidade dos dados que são cedidos pelo sistema que contém as referências sobre os óbitos fetais. Por isso, é primordial captar e entender a prevalência, causas e fatores de risco do óbito fetal para assegurar planos e ações preventivas para impedir complicações e adversidades que poderiam ser evitadas, assim como, mais estudos sobre mortalidade perinatal deveriam ser estimulados uma vez que esses indicadores refletem a qualidade da assistência pré-natal prestada às gestantes (BARROS PDS, et al., 2019).

De acordo com os principais fatores de risco associados ao óbito fetal, identificou-se que a maioria deles se refere às gestantes com transtorno materno hipertensivo (desenvolvimento gradual de hipertensão Pressão arterial maior ou igual a 140×90 mmHg); complicações da placenta; placenta prévia e diabetes. Por outro lado, há uma presença discreta de gestantes com perda fetal prévia e gestantes com o padrão anormal da velocimetria de fluxo das artérias uterinas nas artérias pré-eclâmpticas entre 32 e 37 semanas.

Isso só reflete a importância de uma boa conscientização nas gestantes sobre a relevância do pré-natal e de um bom acompanhamento desde o início da gestação, para que, assim, possa se prevenir possíveis problemas e complicações fetais, acarretando, com isso, em um desenvolvimento fetal sadio e salutar (ANTUNES MB, et al., 2017; MOURA BLA, 2017; PAGE JM e SILVER RM, 2017; REYNA-VILLASMIL E, et al., 2014).

A hipertensão e diabetes foram relacionados às gestantes com idade acima de 35 anos, além de possuírem alguns fatores de risco, tais como: baixo nível socioeconômico, tabagismo, uso de drogas, obesidade e gestantes que tiveram uma má qualidade da assistência pré-natal e escassez no acompanhamento intraparto (ANTUNES MB, et al., 2017; MOURA BLA, 2017; PAGE JM E SILVER RM, 2017). Mulheres com idade de 25 e 44 anos apresentam um indicador marcante na mortalidade fetal, visto que, a idade da mulher está diretamente ligada à TMF. Quanto à escolaridade das mães, os óbitos fetais no Brasil apresentam um crescimento significativo em mulheres com oito anos ou mais de estudo (BARROS PDS, et al., 2019).

Vários artigos trazem o transtorno materno hipertensivo como uma das principais causas da morbimortalidade materna/fetal, por isso, o reconhecimento prévio dessas condições limita o impacto de prováveis problemas e intercorrências gestacionais. Transformar as condutas com ações preventivas e assistenciais pode melhorar e preservar a saúde materno-infantil. Contudo, esse distúrbio traz consigo alguns fatores manifestados como de risco. As condições socioeconômicas e demográficas desfavoráveis (como baixa escolaridade e baixa renda familiar) e o ganho de peso excessivo gestacional são alguns fatores relacionados ao aparecimento desses agravos, levando em consideração que essas circunstâncias estão correlacionadas a decadentes condições nutricionais e obstétricas, assim, acarretando uma gravidez de risco (OLIVEIRA ACMD e GRACILIANO NG, 2015). É relevante pontuar que neste estudo também pudemos observar que a utilização de uma dose de pelo menos $100 \mathrm{mg} /$ dia da aspirina durante a gravidez iniciada antes de 12 semanas pode prevenir a pré-eclâmpsia. Como o transtorno materno hipertensivo é uma das causas mais recorrentes de óbitos fetais, é notável identificar fatores que possam prevenir e/ou evitar que tal transtorno aconteça (BELHOMME N, et al., 2017).

Além disso, foi observado que a utilização do magnésio durante a gravidez não possui efeitos estatisticamente significativos sobre a frequência da mortalidade perinatal, assim como também não houve um resultado considerável sobre a pré-eclâmpsia (MAKRIDES M, et al., 2014).

O tratamento medicamentoso/suplementação é uma maneira muito eficaz na profilaxia da hipertensão gestacional. Os agentes antiplaquetários, quando combinados com cálcio $(2 \mathrm{~g})$, e a suplementação com cálcio 
antes da $20^{\underline{a}}$ semana de gestação produzem uma diminuição significativa da incidência da pré-eclâmpsia (THULER ACDMC, et al., 2018).

O estilo de vida também está fortemente ligado às gestantes que possuem algum transtorno hipertensivo. Sendo assim, a obesidade e o ganho de peso exagerado no decorrer da gravidez aumentam o risco de complicações obstétricas e neonatais. Nesse aspecto, é extremamente importante a implementação de estratégias preventivas e eficazes, como o incentivo às mulheres em idade fértil ao consumo de uma dieta saudável (THULER ACDMC, et al., 2018).

Dois distúrbios hipertensivos são os principais causadores da pressão alta na gestante: a hipertensão crônica e a hipertensão induzida pela gravidez. Também podem ser encontradas a pré-eclâmpsia e a eclâmpsia, essas, por sua vez, apontadas como as causas de mortes obstétricas mais prevalentes (PRADO PF e SILVA SSBED, 2017).

A assistência pré-natal é imprescindível na prevenção do transtorno materno hipertensivo. Torna-se muito importante uma assistência adequada e de qualidade, visto que, a implementação de medidas específicas pode diminuir a mortalidade perinatal e materna (THULER ACDMC, et al., 2018).

Ao refletir acerca da placenta, que é um órgão que se forma dentro do útero durante a gravidez e tem uma importância vital entre a mãe e o bebê por permitir a transferência de nutrientes, oxigênio e contribuir para a descarga de resíduos de sangue do feto, algumas mulheres grávidas podem desenvolver a placenta prévia. Tal problema indica a implantação da placenta na área inferior do útero ou perto do orifício cervical interno. Originalmente, pode ser causada por adesão da placenta, cesarianas, cirurgias uterinas ou acretismo placentário (WALFISCH A e SHEINER E, 2016).

As gestantes que possuem placenta prévia são mais propensas a ter uma placenta que se implanta muito profundamente do útero e acaba não descolando facilmente na hora do parto (placenta acreta). Isto irá acarretar em alto risco de sangramento, necessitando, na maioria das vezes, de uma histerectomia (remoção cirúrgica do útero) como forma de controlar tal hemorragia descompensada. Entre algumas complicações, observa-se sangramento (aproximadamente no segundo trimestre), trabalho de parto prematuro ou natimorto (WALFISCH A e SHEINER E, 2016).

O descolamento prematuro da placenta (DPP) também é reconhecido como uma das principais causas de natimortos, sendo definido como a separação da placenta implantada no corpo do útero, antes do nascimento do feto, em gestação de 20 ou mais semanas completas e resulta de uma série de processos fisiopatológicos, muitas vezes de origem desconhecida. Além de estar associada a grande morbidade materna e perinatal, há maior incidência de anemias, coagulopatias, hemotransfusão, histerectomia e infecções puerperais. A insuficiência uteroplacentária, hemorragia pré-parto e sangramentos vaginais fortemente associado no final da gravidez ou sangramento intraparto também são causas consideradas importantes (AMINU M, et al., 2014).

O diagnóstico do DPP é clínico, descoberto através da sintomatologia e confirmado pela presença de coágulo retroplacentário no pós-parto e placenta com indícios de necrose. A ecografia pode ser útil para um diagnóstico de exclusão de problemas que podem causar sangramento vaginal. Já a tomografia computadorizada (TC) é válida para casos agudos de DPP, sendo também benéfico na ocorrência de traumas (AMINU M, et al., 2014).

A diabetes, por sua vez, considerada um problema de saúde pública, pode ser uma complicação adquirida durante o curso da gravidez. Entre os fatores de risco associados à evolução do diabetes na gestação temos: sobrepeso, multiparidade, feto com malformações genéticas, história de intolerância à glicose, ganho de peso materno maior que $20 \mathrm{~kg}$ em gravidez atual, histórico de condições médicas obstetrícias severas, obesidade, grupo étnico ou raça, idade materna, glicosúria, macrossomia anterior, polidrâmnio anterior, diabetes gestacional e abortos anteriores (MEDINA-PÉREZ EA, et al., 2017).

Com relação à decisão se e quando induzir o parto em gestantes diabéticas depende da idade gestacional, peso fetal estimado e controle glicêmico materno. Pesquisas futuras são necessárias com relação à prevenção do diabetes mellitus gestacional, objetivos de tratamento e eficácia das intervenções, diretrizes para cuidados com a gravidez e prevenção de sequelas metabólicas de longo prazo para o bebê e a mãe. $O$ 
sucesso no tratamento de grávidas com diabetes se dá com um diagnóstico precoce e é baseado no controle glicêmico das pacientes. É fundamental que o cuidado seja rigoroso, tendo em mente todas as complicações e efeitos adversos que a patologia pode acarretar para a saúde materno-infantil, e, principalmente, provocar o óbito fetal (MEDINA-PÉREZ EA, et al., 2017).

Outro fator decisivo para o desenvolvimento de uma gestação de risco é o sobrepeso, pois, mulheres com este problema possuem maior probabilidade de sofrer complicações na gravidez, parto cesáreo, diabetes e hipertensão quando comparadas às mulheres com peso normal. Por outro lado, mulheres com baixo peso possuem maiores chances de nanismo. Dessa forma, o controle de peso é importante para todas as mulheres em idade reprodutiva, além de evitar resultados adversos de nascimento (KHAN MN, et al., 2017).

Porém, é necessário um trabalho de qualidade por parte dos profissionais para estimular as mulheres a ter hábitos saudáveis e cuidarem mais de si, visto que, a autoestima pode estar prejudicada por efeito do aumento de peso. Nesse aspecto, o empoderamento feminino é um fator fundamental na redução das taxas de mortalidade fetal, já que, com isso, elas se consideram mais preparadas a cuidarem de si mesmas, melhorando significativamente o seu pré-natal e também o cuidado intraparto (BARROS PDS, AQUINO ÉCD E SOUZA MRD, 2019).

As manifestações clínicas que as gestantes podem apresentar por morte fetal são: dor de estômago, corrimento vaginal com sangue e/ou aguado, inchaço, diminuição dos movimentos fetais e mal-estar geral (KHAN MN, et al., 2017). Realizar a detecção precoce pode proteger a vida da mãe e do feto, por isso a importância de realizar exames periódicos, como: ultrassom (permite controlar e monitorar o estado da gravidez, bem como visualizar através de imagens a anatomia do feto), ultrassom Doppler (permite detectar anormalidades ou problemas de crescimento da criança). Alguns estudos também indicaram que a prevalência dos anticorpos anticardiolipina $(\mathrm{aCL})$ é alta em uma história de aborto espontâneo recorrente. Esses anticorpos são específicos para os fosfolipídios expressos na membrana celular e contribuem para a perda fetal precoce, pois atuam reduzindo a implantação trofoblástica. Há evidências de que eles afetam aproximadamente $10 \%$ dos casos de aborto espontâneo recorrente (SANTOS NSGM, et al., 2015).

Durante a busca das informações, também foi observado relato de limitações dos estudos pesquisados secundárias à deficiência na qualidade das informações das declarações de óbitos fetais. Sendo assim, é importante a necessidade de orientação por parte dos médicos para o correto e satisfatório preenchimento das declarações de óbitos, principalmente das causas de morte.

\section{CONSIDERAÇÕES FINAIS}

Os fatores de risco mais prevalentes que foram associados aos óbitos fetais foram: transtorno materno hipertensivo, complicações da placenta, placenta prévia e diabetes. Seria interessante a abordagem desta temática em futuros estudos, pois isso pode contribuir com uma melhor compreensão dos fatores relacionados ao óbito fetal atualmente, podendo-se pensar em estratégias mais fidedignas de prevenção. Além disso, é indispensável aperfeiçoar a completitude e determinação das causas de morte dos óbitos fetais, e, também, o desenvolvimento de hábitos que favoreçam o correto fluxo das informações já disponíveis. Por outro lado, a assistência prestada por parte dos profissionais de saúde é primordial para investigar possíveis anormalidades que podem estar acontecendo na gestante, além de conseguir manter a mulher mais orientada quanto à importância da realização de exames necessários, com isso, tendo uma investigação mais apurada de possíveis problemas.

\section{REFERÊNCIAS}

1. AMINU M, et al. Causes of and factors associated with stillbirth in low-and middle-income countries: a systematic literature review. BJOG: An International Journal of Obstetrics \& Gynaecology, 2014; 121, 141-153.

2. ANTUNES MB, et al. Síndrome hipertensiva e resultados perinatais em gestação de alto risco. Rev Reme Enferm, 2017; 21:1057.

3. BARROS PDS, et al. Mortalidade fetal e os desafios para a atenção à saúde da mulher no Brasil. Revista de Saúde Pública, 2019; 53: 12. 
4. BELHOMME N, et al. Aspirine: indications et utilisation durant la grossesse. La revue de médecine interne, $2017 ; 38$ (12): 825-832.

5. COUTINHO EDC, et al. Gravidez e parto: O que muda no estilo de vida das mulheres que se tornam mães?. Revista da Escola de Enfermagem da USP, 2014; 48:17-24.

6. DALLA COSTA L, et al. Perfil epidemiológico de gestantes de alto risco. Cogitare Enfermagem, 2016; 21 (2).

7. ESTRATÉGICAS, Programáticas. Gestação de alto risco: manual técnico. Ministério da Saúde, 2010.

8. GBD 2016 Mortality Collaborators. Global, regional, and national under-5 mortality, adult mortality, age-specific mortality, and life expectancy, 1970-2016: a systematic analysis for the Global Burden of Disease Study 2016. Lancet. 2017;390(10100):1084-150.

9. HOPIA H, et al. Reviewing the methodology of an integrative review. Scandinavian Journal of Caring Sciences, 2016; 30(4): 662-669.

10. KHAN MN, et al. Maternal undernutrition and excessive body weight and risk of birth and health outcomes. Archives of Public Health, 2017; 75 (1): 12.

11. MAKRIDES M, et al. Magnesium supplementation in pregnancy. Cochrane Database of Systematic Reviews, 2014; 4.

12. MEDINA-PÉREZ EA, et al. Diabetes gestacional. Diagnóstico y tratamiento en el primer nivel de atención. Medicina interna de México, 2017; 33(1): 91-98.

13. MOURA BLA. Gestantes usuárias do Sistema Único de Saúde no município de São Paulo: desfechos de uma coorte de dados secundários. Dissertação (Doutorado) - Universidade de São Paulo, 2017.

14. OLIVEIRA ACMD, GRACILIANO NG. Síndrome hipertensiva da gravidez e diabetes mellitus gestacional em uma maternidade pública de uma capital do Nordeste brasileiro, 2013: prevalência e fatores associados. Epidemiologia e Serviços de Saúde, 2015; 24: 441-451.

15. PAGE JM, SILVER RM. Interventions to prevent stillbirth. In Seminars in Fetal and Neonatal Medicine WB Saunders, 2017; 22 (3): 135-145.

16. PRADO PF, SILVA SSBED. Uso da monitoração ambulatorial da pressão arterial nos distúrbios hipertensivos gestacionais. ABCS health sci, 2017; 105-108.

17. REYNA-VILLASMIL E, et al. Doppler velocimetry of the uterine artery blood flow and risk of perinatal death in preeclamptic patients. Clinica e investigation en ginecologia y obstetrician, 2014; 41(4): 158-163.

18. SANTOS NSGM, et al. Anticardiolipin antibodies and recurrent spontaneous abortion in Brazilian women. Scientia Medica, 2015; 25 (1): 2.

19. SIMPSON L, et al. ACR Appropriateness Criteria Assessment of Fetal Well-Being. Journal of the American College of Radiology, 2016; 13 (12): 1483-1493.

20. THULER ACDMC, et al. Medidas preventivas das síndromes hipertensivas da gravidez na atenção primária. Rev. enferm. UFPE on line, 2018; 12 (4): 1060-1071.

21. WALFISCH A, SHEINER E. Placenta previa and immediate outcome of the term offspring. Archives of gynecology and obstetrics, 2016; 294 (4): 739-744. 